\title{
Electron parallel transport for arbitrary collisionality
}

Jeong-Young Ji, Gunsu S. Yun, Yong-Su Na, and Eric D. Held

Citation: Physics of Plasmas 24, 112121 (2017);

View online: https://doi.org/10.1063/1.5004531

View Table of Contents: http://aip.scitation.org/toc/php/24/11

Published by the American Institute of Physics

\section{Articles you may be interested in}

Electric field formation in three different plasmas: A fusion reactor, arc discharge, and the ionosphere

Physics of Plasmas 24, 112505 (2017); 10.1063/1.5002162

Lifetime and universal distribution of seed runaway electrons

Physics of Plasmas 24, 112509 (2017); 10.1063/1.5001931

Emergent kink stability of a magnetized plasma jet injected into a transverse background magnetic field

Physics of Plasmas 24, 110702 (2017); 10.1063/1.5010188

Observation of radially inward turbulent particle flux in ETG dominated plasma of LVPD

Physics of Plasmas 24, 112115 (2017); 10.1063/1.5012093

Ion parallel closures

Physics of Plasmas 24, 022127 (2017); 10.1063/1.4977054

Electron holes in phase space: What they are and why they matter

Physics of Plasmas 24, 055601 (2017); 10.1063/1.4976854

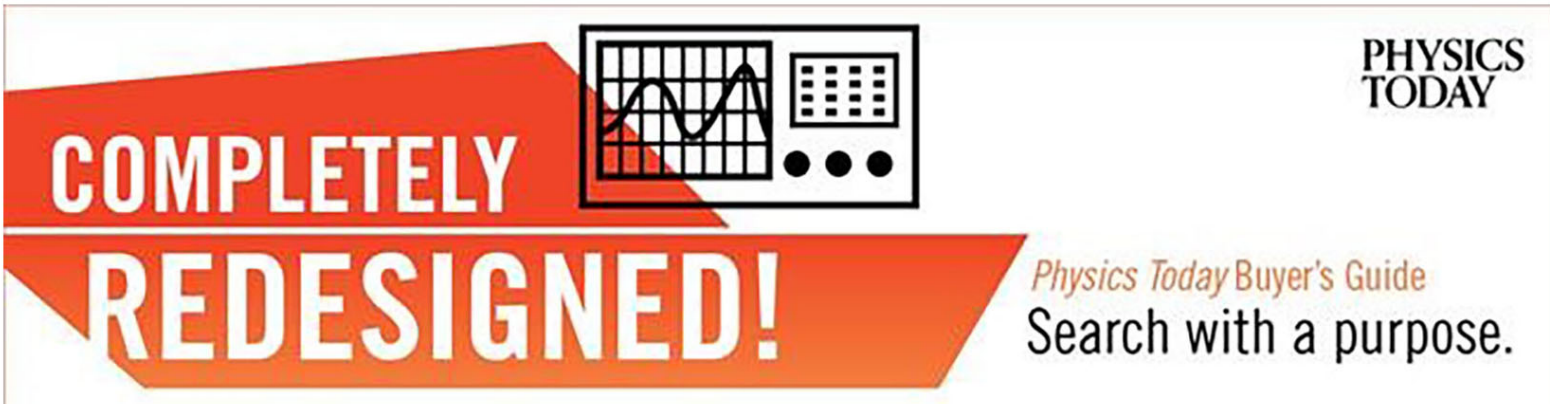




\title{
Electron parallel transport for arbitrary collisionality
}

\author{
Jeong-Young Ji, ${ }^{1, a)}$ Gunsu S. Yun, ${ }^{2}$ Yong-Su Na, ${ }^{3}$ and Eric D. Held ${ }^{1}$ \\ ${ }^{1}$ Department of Physics, Utah State University, Logan, Utah 84322, USA \\ ${ }^{2}$ Department of Physics, Pohang University of Science and Technology, Pohang, Gyeongbuk 790-784, \\ South Korea \\ ${ }^{3}$ Department of Nuclear Engineering, Seoul National University, Seoul 151-742, South Korea
}

(Received 13 September 2017; accepted 7 November 2017; published online 28 November 2017)

\begin{abstract}
Integral (nonlocal) closures [J.-Y. Ji and E. D. Held, Phys. Plasmas 21, 122116 (2014)] are combined with the momentum balance equation to derive electron parallel transport relations. For a single harmonic fluctuation, the relations take the same form as the classical Spitzer theory (with possible additional terms): The electric current and heat flux densities are connected to the modified electric field and temperature gradient by transport coefficients. In contrast to the classical theory, the dimensionless coefficients depend on the collisionality quantified by a Knudsen number, the ratio of the collision length to the angular wavelength. The key difference comes from the proper treatment of the viscosity and friction terms in the momentum balance equation, accurately reflecting the free streaming and collision terms in the kinetic equation. For an arbitrary fluctuation, the transport relations may be expressed by a Fourier series or transform. For low collisionality, the electric resistivity can be significantly larger than that of classical theory and may predict the correct timescale for fast magnetic reconnection. Published by AIP Publishing. https://doi.org/10.1063/1.5004531
\end{abstract}

\section{INTRODUCTION}

In the electron transport theory, the current and heat flux densities are related to the (modified) electric field and temperature gradient. The transport depends on collisionality. The inverse collisionality is often quantified by the Knudsen number $k=\lambda_{\mathrm{C}} / L$, where $\lambda_{\mathrm{C}}$ is the collision length and $L$ is the gradient scale length. For a sinusoidal drive of wavelength $\lambda, L=\lambda / 2 \pi$ and $k=2 \pi \lambda_{\mathrm{C}} / \lambda$. For high-collisionality $(k \ll 1)$ plasmas, the electron parallel transport is described by the Spitzer theory, ${ }^{1,2}$ where the flux densities are connected to thermodynamic drives by transport coefficients. For intermediate to low collisionality, integral (nonlocal) transport effects are considered in Refs. 3-5, where Lorentz model operators are used in the kinetic equation. Lorentz type operators with a momentum restoring term are used to obtain closures and transport also in Refs. 6 and 7. The transport relations can be used to close the electron fluid system of density $(n)$ and temperature $(T)$ when the ion flow velocity is provided by the ion fluid equations. Throughout this paper, the electron species index e is suppressed unless it is needed for clarity.

A Lorentz-type operator is a good approximation for electron-ion collisions and can be used for large ion charge number $Z$. For small $Z$, however, the electron-electron collision operator should be included for accurate evaluation of collision effects. With the electron-electron collision operator included, the electron parallel $(\|)$ closures have been obtained in integral form for arbitrary collisionality in Refs. 8 and 9 . In principle, the parallel viscosity $\left(\pi_{\|}\right)$and the parallel friction force density $\left(R_{\|}\right)$can be incorporated in the electron momentum balance equation

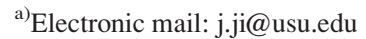

$$
n e E_{\|}+\partial_{\|} p+\partial_{\|} \pi_{\|}=R_{\|}
$$

to find the electron flow velocity $\left(V_{\|}=u\right)$ and subsequently to compute the parallel heat flux density $\left(h_{\|}\right)$from the $h_{\|}$closure relation. In general, this process involves solving an integral equation.

In many interesting problems, ${ }^{3-5,10}$ the small fluctuations and hence the thermodynamic drives are sinusoidal. For a single harmonic drive, the linearized momentum balance Equation (1) becomes algebraic in wave number space. Since the integral closures are expressed by a convolution of a kernel function and a thermodynamic drive in the linearization, the integral closures are algebraic, point-wise multiplication of the kernel and drive, in wave number space. Therefore, the linear system can be solved analytically for a given wave number. In this work, the transport relations for arbitrary collisionality are derived in wave number space. The transport relations have the same form as the classical transport theory ${ }^{1,2,11,12}$ but with dimensionless constant coefficients replaced by $k$-dependent functions. For a general drive, transport can be described by a Fourier series or transform.

In Sec. II, we present the integral closures in configuration space and wave number space where we provide the fitted functions of Fourier transformed kernels. In Sec. III, using the momentum balance equation and closure relations, we obtain transport relations with $k$-dependent transport coefficients. Section IV is devoted to a conclusion.

\section{ELECTRON PARALLEL CLOSURES IN WAVE NUMBER SPACE}

When closing the $\{n, T, \mathbf{V}\}$ fluid moment equations for arbitrary collisionality, the electron parallel closures $\left\{h_{\|}, R_{\|}, \pi_{\|}\right\}$appear in integral form. ${ }^{8}$ For small fluctuations $n=n_{0}+n_{1}, T=T_{0}+T_{1}$ and $\mathbf{V}=\mathbf{V}_{1}$, the linearized closures are written as 


$$
\begin{aligned}
h(\ell)= & -\frac{1}{2} n_{0} v_{0} \int d \eta^{\prime} K_{h h} \frac{\partial T_{1}}{\partial \eta^{\prime}}+p_{0} Z \int d \eta^{\prime} K_{h R} u_{\mathrm{ei}} \\
& -p_{0} \int d \eta^{\prime} K_{h \pi} \frac{\partial u}{\partial \eta^{\prime}} \\
R(\ell)=- & \frac{1}{2} \frac{m v_{0}}{\tau_{\mathrm{ei}}} \frac{n_{0}}{T_{0}} \int d \eta^{\prime} K_{R h} \frac{\partial T_{1}}{\partial \eta^{\prime}} \\
- & \frac{m n_{0}}{\tau_{\mathrm{ei}}}\left(u_{\mathrm{ei}}-Z \int d \eta^{\prime} K_{R R} u_{\mathrm{ei}}\right)-\frac{m n_{0}}{\tau_{\mathrm{ei}}} \int d \eta^{\prime} K_{R \pi} \frac{\partial u}{\partial \eta^{\prime}}, \\
\pi(\ell)= & -n_{0} \int d \eta^{\prime} K_{\pi h} \frac{\partial T_{1}}{\partial \eta^{\prime}}+2 \frac{n_{0} T_{0}}{v_{0}} Z \int d \eta^{\prime} K_{\pi R} u_{\mathrm{ei}} \\
& -\frac{n_{0} T_{0}}{v_{0}} \int d \eta^{\prime} K_{\pi \pi} \frac{\partial u}{\partial \eta^{\prime}} .
\end{aligned}
$$

Here, $\ell$ is the arc-length along a magnetic field line (or a parallel direction $\|), \eta=\ell / \lambda_{\mathrm{C}}\left(\eta^{\prime}=\ell^{\prime} / \lambda_{\mathrm{C}}\right), \lambda_{\mathrm{C}}=v_{0} \tau_{\mathrm{ee}}$ is the collision length, $v_{0}=\sqrt{2 T_{0} / m}$ is the thermal speed, $\tau_{\mathrm{ee}}$ is the electron-electron collision time, $\tau_{\mathrm{ei}}=\tau_{\mathrm{ee}} / Z$ is the electron-ion collision time, $u_{\mathrm{ei}}=V_{\mathrm{e} 1 \|}-V_{\mathrm{i} 1 \|}$ is the relative electron-ion parallel velocity, and $u=V_{\mathrm{e} 1 \|}$. Although fitted kernels for $Z=1$ only will be provided in this work, the ion charge number $Z$ is kept for future purpose. For simplicity, we have assumed $\nabla \cdot \mathbf{V}_{1 \perp}=0$, which is valid for the diamagnetic flow $\mathbf{V}_{1 \perp}=\mathbf{B} \times \nabla p_{0} / q B^{2}$ in slab geometry. When $\nabla \cdot \mathbf{V}_{1 \perp} \neq 0$, the $\partial u / \partial \eta^{\prime}$ terms should be replaced by $\partial u / \partial \eta^{\prime}-\frac{1}{2} \lambda_{\mathrm{C}} \nabla \cdot \mathbf{V}_{1 \perp}$ which becomes $(3 / 2) \partial u / \partial \eta^{\prime}$ when $\nabla \cdot \mathbf{V}_{1 \perp}=-\partial_{\|} u\left(\nabla \cdot \mathbf{V}_{1}\right.$ $=0)$. The fitted kernel functions $K_{A B}\left(\eta-\eta^{\prime}\right)$ are presented in Refs. 8 and 9.

For the Fourier transform and its inverse, $\tilde{A}(k)$ and $A(\eta)$, we adopt the convention: $\tilde{A}(k)=\int d \eta A(\eta) e^{-i k \eta}$ and $A(\eta)$ $=(2 \pi)^{-1} \int d k e^{i k \eta} \tilde{A}(k)$. The Fourier transform of $\int d \eta^{\prime} K_{A B}$ $\left(\eta-\eta^{\prime}\right) g_{B}\left(\eta^{\prime}\right)$ is $\tilde{K}_{A B}(k) \tilde{g}_{B}(k)$ by the convolution theorem. Then, Fourier transforming Eqs. (2)-(4), we obtain closures in wave number space (closures for a single-mode sinusoidal drive):

$$
\begin{aligned}
\tilde{h}_{\|}= & -\frac{1}{2} n_{0} v_{0} \tilde{K}_{h h} i k \tilde{T}+p_{0} Z \tilde{K}_{h R} \tilde{u}_{\mathrm{ei}}-p_{0} \tilde{K}_{h \pi} i k \tilde{u}, \\
\tilde{R}_{\|}= & -\frac{1}{2} \frac{m v_{0}}{\tau_{\mathrm{ei}}} \frac{n_{0}}{T_{0}} \tilde{K}_{R h} i k \tilde{T}-\frac{m n_{0}}{\tau_{\mathrm{ei}}}\left(1-Z \tilde{K}_{R R}\right) \tilde{u}_{\mathrm{ei}} \\
& -\frac{m n_{0}}{\tau_{\mathrm{ei}}} \tilde{K}_{R \pi} i k \tilde{u}, \\
\tilde{\pi}= & -n_{0} \tilde{K}_{\pi h} i k \tilde{T}+2 \frac{p_{0}}{v_{0}} Z \tilde{K}_{\pi R} \tilde{u}_{\mathrm{ei}}-\frac{p_{0}}{v_{0}} \tilde{K}_{\pi \pi} i k \tilde{u},
\end{aligned}
$$

where $\tilde{T}$ is the Fourier transform of $T_{1}$.

The Fourier transformed kernels $\tilde{K}_{A D}(A, D=h, R$, and $\pi$ ) can be compared to the cosine or sine Fourier transform $\hat{K}_{A D}$ in Ref. 8. From Eq. (7) of Ref. 8, we rewrite

$$
\int K_{A D}\left(\eta-\eta^{\prime}\right) \exp \left(i k \eta^{\prime}\right) d \eta^{\prime}= \begin{cases}\hat{K}_{A D} \exp (i k \eta), & \text { for even } \\ \hat{K}_{A D}(-i) \exp (i k \eta), & \text { for odd, }\end{cases}
$$

where even means $A D=h h, h R, R R$, and $\pi \pi$ and odd means $A D=h \pi$, and $R \pi$. Since $K_{A D}$ is real, $\hat{K}_{A D}$ is real and related to $\tilde{K}_{A D}$ as

$$
\hat{K}_{A D}= \begin{cases}\tilde{K}_{A D}, & \text { for even } \\ i \tilde{K}_{A D}, & \text { for odd }\end{cases}
$$

which follows from the comparison between Eq. (8) for $\eta=0$ and the Fourier transform $\int K_{A D}(\eta) e^{-i k \eta} d \eta=\tilde{K}_{A D}(k)$. Therefore, the real closure quantities defined in Ref. 9 are related to the Fourier transformed kernels: $\hat{h}_{h}=k \hat{K}_{h h}, \hat{h}_{R}$ $=Z \hat{K}_{h R}=\hat{R}_{h}, \hat{h}_{\pi}=k \hat{K}_{h \pi}=\hat{\pi}_{h}, \hat{R}_{R}=1-Z \hat{K}_{R R}, \hat{R}_{\pi}=Z \hat{K}_{R \pi}$ $=\hat{\pi}_{R}$, and $\hat{\pi}_{\pi}=k \hat{K}_{\pi \pi}$. Using these dimensionless closures $\hat{A}_{D}$, we rewrite Eqs. (5)-(7) as follows:

$$
\begin{gathered}
\tilde{h}_{\|}=-\frac{1}{2} n_{0} v_{0} \hat{h}_{h} i \tilde{T}+p_{0} \hat{h}_{R} \tilde{u}_{\mathrm{ei}}-p_{0} \hat{h}_{\pi} \tilde{u}, \\
\tilde{R}_{\|}=-\frac{1}{2} \frac{m v_{0}}{\tau_{\mathrm{ee}}} \frac{n_{0}}{T_{0}} \hat{R}_{h} i k \tilde{T}-\frac{m n_{0}}{\tau_{\mathrm{ei}}} \hat{R}_{R} \tilde{u}_{\mathrm{ei}}-\frac{m n_{0}}{\tau_{\mathrm{ee}}} \hat{R}_{\pi} k \tilde{u}, \\
\tilde{\pi}_{\|}=-n_{0} \hat{\pi}_{h} \tilde{T}-2 \frac{p_{0}}{v_{0}} \hat{\pi}_{R} i \tilde{u}_{\mathrm{ei}}-\frac{p_{0}}{v_{0}} \hat{\pi}_{\pi} i \tilde{u} .
\end{gathered}
$$

These closure relations can be used for general drives represented by a Fourier series or spectrum in the limit of small fluctuations.

The Fourier transform of the kernels is obtained from the 6400 moment solutions for $k \leqq 80$ (convergent regime of the moment system $)^{8}$ and from the collisionless-limit solution. ${ }^{13}$ They can be fitted to

$$
\hat{K}_{A B}=\frac{a k^{\alpha}}{1+d_{1} k^{\delta}+d_{2} k^{2 \delta}+d_{3} k^{3 \delta}+d_{4} k^{4 \delta}+d_{5} k^{5 \delta}+d_{6} k^{6 \delta}}
$$

within 3\% error for $k \leqq 80$. The fitted parameters are given in Table I. For $k \gtrsim 80$, the kernels are constructed to monotonically approach asymptotic values. When an asymptotic value is nonzero, the error is still expected to be less than $3 \%$. When an asymptotic value is zero, the percent error could be large, but the corresponding closure value is negligible in the fluid system. Therefore, the fitted kernels are accurate for the entire regime of collisionality. Finally, it should be emphasized that even kernels are proportional to $1 /|k|$ and odd kernels are proportional to $1 / k$ in the collisionless limit $(|k| \rightarrow \infty)$.

\section{ELECTRON PARALLEL TRANSPORT}

Now, we use the closure relations to derive the transport relations. In transport theory, the particle (charge) and heat flux densities are related to thermodynamic drives: The

TABLE I. Fitted parameters for real kernels (13) for $k \geq 0$. The maximum errors are assessed by the 6400 moment closures in the convergent regime. The kernels for $k<0$ can be obtained from $\hat{K}_{A B}(-k)=\hat{K}_{A B}(k)$ for $A B=h h$, $h R, R R$, and $\pi \pi$ and $\hat{K}_{A B}(-k)=-\hat{K}_{A B}(k)$ for $A B=h \pi$ and $R \pi$.

\begin{tabular}{ccccccccccc}
\hline \hline & $a$ & $\alpha$ & $\Delta$ & $d_{1}$ & $d_{2}$ & $d_{3}$ & $d_{4}$ & $d_{5}$ & $d_{6}$ & err. $(\%)$ \\
\hline$\hat{K}_{h h}$ & 3.20 & 0 & $1 / 6$ & 0.675 & -2.65 & 1.30 & 1.97 & -0.279 & 1.58 & 2.9 \\
$\hat{K}_{h R}$ & 0.703 & 0 & $1 / 6$ & 0 & 0.837 & -4.94 & 8.43 & -4.33 & 0.824 & 2.7 \\
$\hat{K}_{h \pi}$ & 1.83 & 1 & $1 / 3$ & 1.497 & -9.36 & 21.7 & -12.9 & 4.11 & 4.575 & 1.3 \\
$\hat{K}_{R R}$ & 0.494 & 0 & $1 / 6$ & 0.0648 & -0.431 & 0.670 & -0.198 & 0.0347 & 0 & 1.0 \\
$\hat{K}_{R \pi}$ & 0.284 & 1 & $1 / 3$ & 0.387 & -2.67 & 6.71 & -3.39 & 2.06 & 0 & 1.5 \\
$\hat{K}_{\pi \pi}$ & 0.978 & 0 & $1 / 6$ & 0 & 0.337 & -1.37 & 1.35 & -0.375 & 0.690 & 0.8 \\
\hline \hline
\end{tabular}


modified electric field and temperature gradient. They can be obtained from the momentum balance equation with closures. The momentum balance Eq. (1) can be linearized as

$$
n_{0} e E_{\|}^{\prime}+\partial_{\|} \pi_{\|}=R_{\|},
$$

where the modified electric field is defined as

$$
E_{\|}^{\prime}=E_{1 \|}+\frac{1}{n_{0} e} \partial_{\|} p_{1} .
$$

While Eq. (14), when combined with Eqs. (3) and (4), is an integro-differential equation in configuration space, it becomes an algebraic equation in wave number space

$$
n_{0} e \tilde{E}_{\|}^{\prime}+i k_{\ell} \tilde{\pi}_{\|}=\tilde{R}_{\|},
$$

where $k_{\ell}=2 \pi / \lambda=k / \lambda_{\mathrm{C}}$. We solve this equation by replacing $\tilde{R}_{\|}$and $\tilde{\pi}_{\|}$with Eqs. (11) and (12) to express $\tilde{u}$ in terms of $\tilde{E}_{\|}^{\prime}$ and $i k_{\ell} \tilde{T}$.

Since the closure relations involve the ion flow velocity $u_{\mathrm{i}}$ in $u_{\mathrm{e}}$, we need the ion momentum balance equation to find it. However, we can show that $u_{\mathrm{i}} \ll u_{\mathrm{e}}$ unless $\partial_{\|} p_{\mathrm{i}}$ $\gg \partial_{\|} p_{\mathrm{e}}, \partial_{\|} T_{\mathrm{i}} \gg \partial_{\|} T_{\mathrm{e}}$ or $T_{\mathrm{i}} \gg T_{\mathrm{e}}$. Therefore, we approximate $u_{\mathrm{ei}} \approx u_{\mathrm{e}}=u$ and obtain

$$
\tilde{u}=\frac{\tau_{\mathrm{ei}}}{m} \frac{\left(\hat{h}_{\pi}-\hat{h}_{R}\right) i k_{\ell} \tilde{T}-e \tilde{E}_{\|}^{\prime}}{\Delta},
$$

where

$$
\Delta=\frac{k}{Z}\left(\frac{1}{2} \hat{\pi}_{\pi}+2 \hat{R}_{\pi}\right)+\hat{R}_{R}
$$

With Eq. (17) obtained, it follows from $J_{\|}=-n_{0} e u$ and Eq. (10) that

$$
\begin{aligned}
& \tilde{J}_{\|}=\sigma_{\|} \tilde{E}_{\|}^{\prime}-\alpha_{\|} i k_{\ell} \tilde{T}, \\
& \tilde{h}_{\|}=\alpha_{\|} \tilde{E}_{\|}^{\prime}-\kappa_{\|} i k_{\ell} \tilde{T},
\end{aligned}
$$

where

$$
\begin{gathered}
\sigma_{\|}=\frac{n_{0} e^{2} \tau_{\mathrm{ei}}}{m} \tilde{\sigma}_{\|}, \quad \tilde{\sigma}_{\|}=\frac{1}{\Delta}, \\
\alpha_{\|}=\frac{n_{0} e \tau_{\mathrm{ei}}}{m} \tilde{\alpha}_{\|}, \quad \tilde{\alpha}_{\|}=\frac{\hat{h}_{\pi}-\hat{h}_{R}}{\Delta}, \\
\kappa_{\|}=\frac{n_{0} T_{0} \tau_{\mathrm{ei}}}{m} \tilde{\kappa}_{\|}, \quad \tilde{\kappa}_{\|}=Z \frac{\hat{h}_{h}}{k}+\frac{\left(\hat{h}_{\pi}-\hat{h}_{R}\right)^{2}}{\Delta} .
\end{gathered}
$$

Note that the transport relations (19) and (20) for a single harmonic drive appear in classical form except that the dimensionless transport coefficients (with a tilde) depend on $k$. In the classical theory for high collisionality, they are constants.

The dimensionless transport coefficients are plotted in Fig. 1. In the collisional limit $(k \rightarrow 0)$, the coefficients reproduce the classical transport coefficients: $:^{1,2,11} \tilde{\sigma}_{\|} \rightarrow 1.98$ $\simeq \tilde{\sigma}_{\|}^{\mathrm{LS}}, \tilde{\alpha}_{\|} \rightarrow-1.39$, and $\tilde{\kappa}_{\|} \rightarrow 4.18$ which agree with the convergent values from the moment approach. ${ }^{12}$ In the collisionless limit $(k \rightarrow \pm \infty), \tilde{\sigma}_{\|} \rightarrow 1.41 /|k|, \tilde{\alpha}_{\|} \rightarrow 0.564 /|k|$,
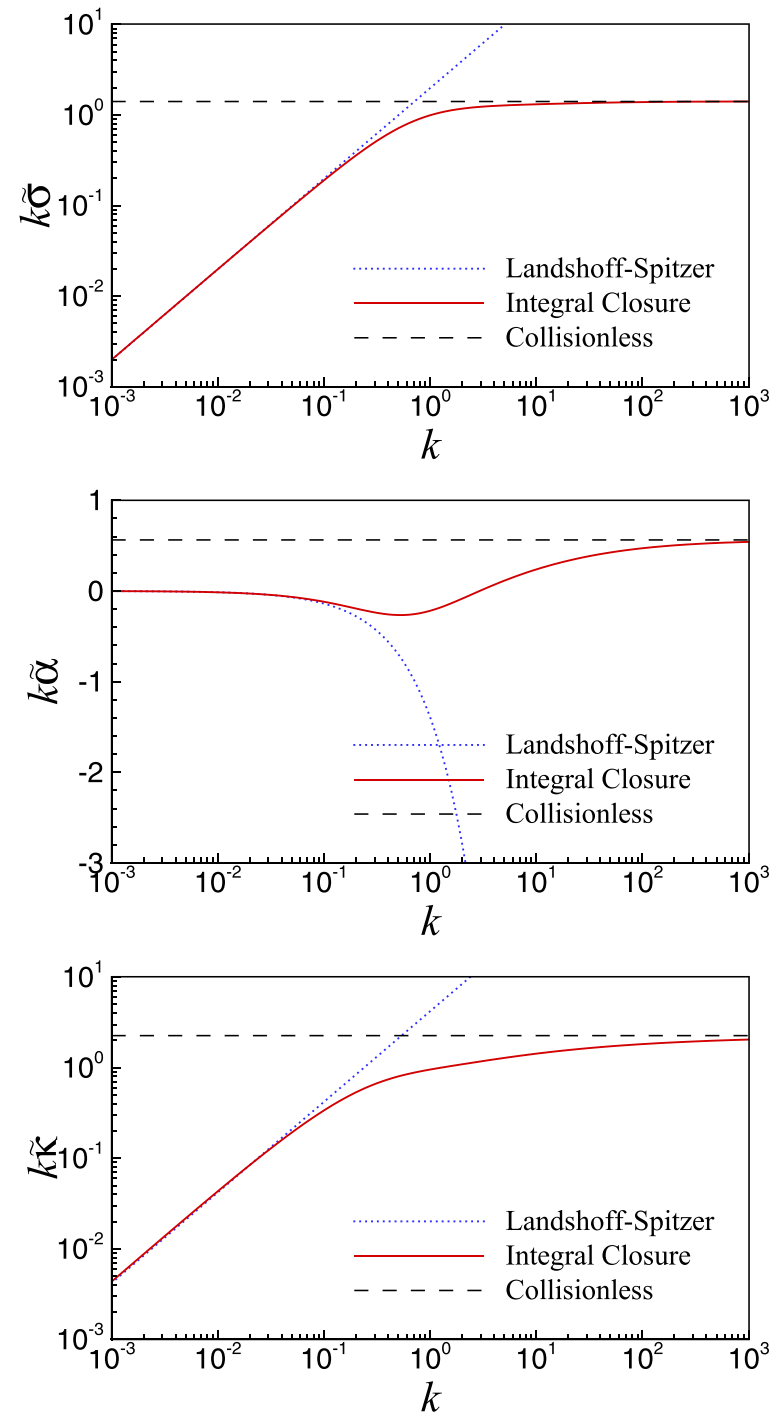

FIG. 1. Transport coefficients: Eqs. (21)-(23) for sinusoidal drives are plotted from the integral closures (red, solid). Landshoff-Spitzer's collisional (blue, dotted) and the collisionless (black, dashed) limits are also shown.

and $\quad \tilde{\kappa}_{\|} \rightarrow 2.26 /|k|$ where the coefficients agree with the theoretical values in Ref. 13: $5 / 2 \sqrt{\pi} \approx 1.41,1 / \sqrt{\pi}$ $\approx 0.564$, and $4 / \sqrt{\pi} \approx 2.26$. Note that $\tau_{\mathrm{ei}} /|k|=\lambda / 2 \pi v_{0} Z$ in the transport coefficients (21-23). For a given wavelength of fluctuation $\lambda$, they are proportional to $T^{-1 / 2}$ at low collisionality.

In the derivation of Eqs. (19) and (20), the $\mathbf{V}_{\perp}$ terms have been ignored. One of them is in the rate of strain tensor and modifies the $\partial u / \partial \eta^{\prime}$ terms to $\partial u / \partial \eta^{\prime}-\frac{1}{2} \lambda_{\mathrm{C}} \nabla \cdot \mathbf{V}_{1 \perp}$ in the closure Equations (2-4). This introduces an additional term into Eqs. (19) and (20), respectively,

$$
\tilde{J}_{\gamma}=-n_{0} e \frac{k\left(\hat{\pi}_{\pi} / 2+\hat{R}_{\pi}\right)}{2 Z \Delta} \frac{\tilde{\gamma}}{i k}
$$

and

$$
\tilde{h}_{\gamma}=\frac{p_{0} \hat{h}_{\pi}}{2} \frac{\tilde{\gamma}}{i k},
$$

where $\tilde{\gamma}=\int d \eta e^{-i k \eta} \lambda_{\mathrm{C}} \nabla \cdot \mathbf{V}_{1 \perp}=\int d \ell e^{-i k_{\ell} \ell} \nabla \cdot \mathbf{V}_{1 \perp}$. In the collisional limit, $\tilde{J}_{\gamma} \rightarrow 0$ and $\tilde{h}_{\gamma} \rightarrow 0$ as expected. In the 
collisionless limit, $\tilde{J}_{\gamma} \rightarrow-n_{0} e \tilde{\gamma} / 2 i k$ and $\tilde{h}_{\gamma} \rightarrow p_{0} \tilde{\gamma} / 5 i k$ (they become integration in configuration space). There may be additional modification from the advection term $(\mathbf{V} \cdot \nabla \mathbf{V})_{\|}$ and the gyro-viscosity term $\left(\nabla \cdot \pi^{\mathrm{gv}}\right)_{\|}$in the momentum balance equation. These terms may be accurately treated in a fluid code which evolves the momentum equation.

For arbitrary collisionality, Ohm's law $J_{\|}=\sigma_{\|} E_{\|}$can be obtained from the solution of the reduced kinetic equation

$$
v_{\|} \partial_{\|} f_{1}-C f_{1}=-\frac{e}{m} E_{\|} \frac{\partial f_{0}}{\partial v_{\|}},
$$

with $f_{0}$ being a static Maxwellian distribution. In wave number space, the solution is

$$
\tilde{f}_{1}=\frac{e}{m} \tilde{E}_{\|} \frac{\partial f_{0} / \partial v_{\|}}{C-v_{\|} i k_{\ell}}=\frac{e}{m} \frac{C+v_{\|} i k_{\ell}}{C^{2}+\left(v_{\|} k_{\ell}\right)^{2}} \frac{\partial f_{0}}{\partial v_{\|}} \tilde{E}_{\|} .
$$

The key difference from Spitzer theory is the inclusion of the free streaming term $v_{\|} \partial f_{1}$. Without the streaming term, the electric conductivity increases (resistivity decreases) linearly as the collision time increases ( $C$ decreases) linearly. The streaming term, however, plays the role of an effective collision operator, significantly reducing the conductivity (raising the resistivity) at low collisionality. In the general moment method, we evaluate the collision and streaming terms accurately to obtain closures and use them in the momentum balance equation. The kinetic effects are well captured by $R_{\|}$ and $\partial_{\|} \pi_{\|}$, a moment of the streaming term. At low collisionality, the major contribution to the conductivity (resistivity) comes from the parallel viscosity closure driven by the flow velocity gradient, $\hat{\pi}_{\pi}$ term in Eq. (18).

It should be emphasized that, as $k$ becomes large, $k \tilde{\sigma}_{\|}$ becomes constant, while it is proportional to $k$ in the Spitzer theory. For a given wavelength of spatial fluctuation, it follows from $\tau_{\text {ei }} \propto T^{3 / 2}$ and $v_{0} \propto T^{1 / 2}$ that $\sigma_{\|} \propto T^{-1 / 2}$, while $\sigma_{\|}^{\mathrm{LS}} \propto T^{3 / 2}$ in the Spitzer theory. This will modify the resistivity in the generalized Ohm law. Among many applications, we note that the magnetic diffusion and the magnetic reconnection time are determined by $\tau_{\text {rec }}=\mu_{0} \sigma_{\|}\left|\nabla^{2}\right|^{-1}$ (see e.g., Ref. 14 for general discussion). Therefore, the time scale of this work is significantly shorter than that of Spitzer theory. The quantitative ratio is

$$
\frac{\tau_{\text {rec }}}{\tau_{\text {rec }}^{\mathrm{LS}}}=\frac{\sigma_{\|}}{\sigma_{\|}^{\mathrm{LS}}} \stackrel{k \rightarrow \infty}{\longrightarrow} \frac{1}{1.4 k}=\frac{1}{8.8} \frac{\lambda}{\lambda_{\mathrm{C}}},
$$

where we note that $\lambda_{\mathrm{C}} \propto T^{2}$. The discrepancy is greater at higher temperature (lower collisionality) for given density and wavelength. This implies that spatial inhomogeneity enhances fast magnetic reconnection when the gradient scale length is much smaller than the collision length. The magnetic reconnection process is faster at higher temperature (low collisionality) and with a shorter wavelength of fluctuation (gradient scale length).

\section{CONCLUSION}

Electron parallel transport relations are derived from integral closures for arbitrary collisionality. The relations make considerable modification to the Spitzer theory for low collisionality. The modification is mainly due to the accurate evaluation of the kinetic response in deriving the parallel viscosity closure driven by the flow velocity gradient $\left[\hat{\pi}_{\pi}\right.$ in Eq. (18)]. The transport relations derived here will evaluate parallel electric current and heat flux densities accurately for arbitrary collisionality in the low-frequency, linear regime.

Many plasma fluid codes evolve the momentum (flow velocity) evolution equation without imposing adiabatic and linear approximations on the equation. Since the major modification originates from the viscosity term, adopting the integral closures introduces the effects of modified transport relations with the nonlinear and non-adiabatic terms being kept. In particular, the closure relations in wave number space can be conveniently implemented in BOUT $++{ }^{15}$ using the fast non-Fourier method. ${ }^{16}$ In NIMROD ${ }^{17}$ simulations, the electric resistivity with a representative wave number can be used in the generalized Ohm law with or without Hall, advection, inertia, terms, etc. For a given wave number $k$ and angular frequency $\omega$, the effects of inertia and nonlinear terms in the momentum equation can be evaluated by the Fourier expansion method. The inertia term will introduce a $-i \omega$ factor into the equation. The nonlinear advection term, $u \partial_{\|} u$, for example, will introduce coupling terms between different wave numbers $\sum_{k^{\prime}} u_{k^{\prime}} u_{k-k^{\prime}}$ into the $k$ mode equation. This will further modify the transport coefficients. The Fourier method will be used to analyze the results of numerical simulations.

\section{ACKNOWLEDGMENTS}

The research was supported by the U.S. DOE under Grant Nos. DE-SC0014033, DE-SC0016256, DE-FC0208ER54973, and DE-FG02-04ER54746. This work was performed in conjunction with the Plasma Science and Innovation (PSI) center and the Center for Extended MHD Modeling (CEMM).

${ }^{1}$ R. Landshoff, Phys. Rev. 76, 904 (1949).

${ }^{2}$ L. Spitzer, Jr. and R. Härm, Phys. Rev. 89, 977 (1953).

${ }^{3}$ A. R. Bell, Phys. Fluids 26, 279 (1983).

${ }^{4}$ E. M. Epperlein, Phys. Rev. Lett. 65, 2145 (1990).

${ }^{5}$ E. M. Epperlein, R. W. Short, and A. Simon, Phys. Rev. Lett. 69, 1765 (1992).

${ }^{6}$ Z. Chang and J. D. Callen, Phys. Fluids B 4, 1167 (1992).

${ }^{7}$ Z. Chang and J. D. Callen, Phys. Fluids B 4, 1182 (1992).

${ }^{8}$ J.-Y. Ji and E. D. Held, Phys. Plasmas 21, 122116 (2014); 22, 129901 (2015).

${ }^{9}$ J.-Y. Ji, S.-K. Kim, E. D. Held, and Y.-S. Na, Phys. Plasmas 23, 032124 (2016).

${ }^{10}$ J. R. Albritton, Phys. Rev. Lett. 50, 2078 (1983).

${ }^{11}$ R. Balescu, Transport Processes in Plasmas (North-Holland, Amsterdam, 1988), Vol. 1

${ }^{12}$ J.-Y. Ji and E. D. Held, Phys. Plasmas 20, 042114 (2013).

${ }^{13}$ J.-Y. Ji, E. D. Held, and H. Jhang, Phys. Plasmas 20, 082121 (2013).

${ }^{14}$ D. Biskamp, Magnetic Reconnection in Plasmas, 2nd ed. (Cambridge University Press, Cambridge, 2000).

${ }^{15}$ B. D. Dudson, M. V. Umansky, X. Q. Xu, P. B. Snyder, and H. R. Wilson, Comput. Phys. Commun. 180, 1467 (2009).

${ }^{16}$ A. M. Dimits, I. Joseph, and M. V. Umansky, Phys. Plasmas 21, 055907 (2014).

${ }^{17}$ C. R. Sovinec, A. H. Glasser, T. A. Gianakon, D. C. Barnes, R. A. Nebel, S. E. Kruger, D. D. Schnack, S. J. Plimpton, A. Tarditi, M. S. Chu, and the NIMROD Team. J. Comput. Phys. 195, 355 (2004). 